\title{
Investigation of the relationship between cord clamping time and risk of hyperbilirubinemia
}

\author{
Yüksel Yaşartekin ${ }^{1 \oplus}$, S.Ümit Sarıc1 ${ }^{1 \oplus}$, Murat Özcan ${ }^{2 \oplus}$, Melis Akpınar ${ }^{1 \oplus}$, \\ Demet Altun ${ }^{1 \oplus}$, Agah Akın ${ }^{1 \oplus}$, Muhittin A. Serdar ${ }^{3 \oplus}$, Dilek Sarıc1 ${ }^{4 \oplus}$ \\ ${ }^{1}$ Division of Neonatology, Department of Pediatrics, Ufuk University Faculty of Medicine, Ankara; ${ }^{2}$ Department of Pediatrics, \\ State Hospital, Haymana, Ankara; ${ }^{3}$ Department of Biochemistry, Acıbadem University Faculty of Medicine, Ankara; ${ }^{4}$ Division of \\ Neonatology, Department of Pediatrics, Kecioren Education and Research Hospital, Ankara, Turkey.
}

\begin{abstract}
Background. Although the relationship between umbilical cord clamping time and various parameters such as hemoglobin $(\mathrm{Hb})$ levels, iron deficiency, and risk of neonatal jaundice has previously been studied, to the best of our knowleadge there have been no studies investigating the relationship between cord clamping time and the risk of significant hyperbilirubinemia. We aimed to investigate the relationship between the time of umbilical cord clamping and transcutaneous bilirubin $(\mathrm{TcB})$ measurements made on various postnatal hours, $\mathrm{Hb}$ and serum total bilirubin (STB) levels measured on postnatal 4th day, and the risk of development of significant hyperbilirubinemia requiring phototherapy treatment.
\end{abstract}

Methods. Eligible newborns were divided into two groups on the basis of the time of cord clamping: those clamped late (60 seconds or more; Group I) and those clamped early (less than 60 seconds; Group II). Groups were compared with respect to the parameters of cord $\mathrm{Hb}$, postnatal TcB measurements at 6th, 48th, 96th and 168th hours, and 96th hour $\mathrm{Hb}$, STB and direct bilirubin levels.

Results. TcB levels at the 96th and 168th hour were significantly higher in Group I when compared to Group II $(p<0.001$ and $p<0.001$, respectively). The 96th hour STB level was significantly higher in Group I when compared to Group II ( $\mathrm{p}<0.001)$. The need of phototherapy requirement was higher in Group I when compared to Group II ( $\mathrm{p}=0.001$ ). Increase in cord blood $\mathrm{Hb}$ for each $1 \mathrm{gr} / \mathrm{dl}$ caused a 3.94-fold increased risk in the requirement of phototherapy treatment. Cord clamping time showed statistically significant positive correlations with both cord blood and 96th hour venous Hb levels, with both 96th hour and 168th hour TcB levels, and with 96th hour STB levels.

Conclusions. Newborns whose cords are clamped late should be followed up closely with respect to high postnatal bilirubin levels and other risks associated with significant hyperbilirubinemia requiring phototherapy treatment.

Key words: hyperbilirubinemia; risk management; transcutaneous bilirubin measurements.

One of the most serious issues concerning the risk of the development of significant hyperbilirubinemia is the timing of umbilical cord clamping. International Liaison Committee

$\bowtie$ Demet Altun

draltundemet@gmail.com

Received: 9th May 2019, revised 13th September 2019, 4th November 2019, accepted 10th November 2019.

The study was presented as a poster presentation at the 26th National Neonatology Congress (UNEKO-26) on April 14-18, 2018, Cyprus.
On Resuscitation-Consensus on Science with Treatment Recommendations (ILCOR-CoSTR) recommends delaying cord clamping by at least 60 seconds in newborns who do not require resuscitation. ${ }^{1}$ Such a delayed cord clamping provides the newborn an additional blood volume of at least $30 \%$ and an additional erythrocyte mass up to $60 \% \cdot^{2-4}$ On the other hand higher umbilical cord hemoglobin $(\mathrm{Hb})$ levels due to delayed cord clamping have caused an increased incidence of significant hyperbilirubinemia requiring phototherapy, 
and each 1 gram increase in cord $\mathrm{Hb}$ levels has increased phototherapy requirement by 1.7 times in a study conducted in healthy newborns. ${ }^{5}$

Although there is only one study investigating the relationship between cord blood parameters $(\mathrm{Hb}$ level) and predicting the risk of later developing significant hyperbilirubinemia in the era of early discharge from the hospital, ${ }^{5}$ there is, to our knowledge, no study investigating the relationship between the time of umbilical cord clamping and the risk of significant hyperbilirubinemia development especially with both serum total bilirubin (STB) and transcutaneous bilirubin (TcB) measurements. We, thus, aimed to investigate the relationship between the time of umbilical cord clamping and TcB measurements made on various postnatal hours, $\mathrm{Hb}$ and STB levels measured on postnatal 4th day, and the risk of development of significant hyperbilirubinemia requiring phototherapy treatment.

\section{Material and Methods}

The study was conducted at the Division of Neonatology, Department of Pediatrics of Ufuk University Faculty of Medicine between May 2016 and May 2017. Cord blood samples were obtained from all term and late preterm newborns born during the study period.

Umbilical cords of newborns who late clamping was performed were clamped and cut holding the cord on the level with placenta for 6090 seconds after birth, and those who were clamped early were clamped and cut at a distance of 2-3 cm near to umbilicus within 60 seconds. Milking was not performed in early cord clamping. Complete blood count, blood group and direct antiglobulin (Coombs) tests were studied from the samples taken from the cord blood. TcB measurements were made at the 6th, 48th, 96th and 168th hours, and venous $\mathrm{Hb}$, STB and direct bilirubin levels were measured at the 96th hour in order to determine the risk of significant hyperbilirubinemia and investigate the relationship between umbilical cord $\mathrm{Hb}$ and postnatal bilirubin levels. Patients were divided into two groups on the basis of the time of cord clamping: those clamped late $(60$ seconds or more; Group I) and those clamped early (Less than 60 seconds; Group II). Groups were compared with respect to the parameters of cord $\mathrm{Hb}$, postnatal $\mathrm{TcB}$ measurements at $6 \mathrm{th}$, 48th, 96th and 168th hours, 96th hour $\mathrm{Hb}$, STB and direct bilirubin levels, gender, gestational age, route of delivery, birth weight, Apgar scores at 1 st and 5 th minutes, maternal age, number and ratio of late preterm newborns and weight loss until postnatal 4th day as grams and per cent. Correlation analysis between the time of cord clamping and all the other parameters were performed for all cases included (Group I+Group II).

Newborns with a gestational age of $\geq 35$ weeks were included in the study. As prematurity itself is a major risk factor in the etiology of hyperbilirubinemia, preterm newborns with a gestational age of $<35$ weeks were not included in the study. Other exclusion criteria were newborns with isoimmunization $(\mathrm{ABO}, \mathrm{Rh}$ or subgroup incompatibilities), direct Coombs test positivity, findings of hemolysis on blood smear (anisocytosis, spherocytosis, polychromasia, poikilocytosis), anemia, reticulocytosis and/or glucose-6-phosphate dehydrogenase deficiency, the presence of any major congenital anomaly, respiratory distress, pathologic weight loss of $>10 \%$, the need of resuscitation at birth and clinical or culture-proven sepsis.

In all cases in the study, gender, birth weight, route of delivery, maternal age, gestational age, Apgar scores at 1st and 5th minutes, blood groups and $\mathrm{Rh}$ types in mother-infant pairs, umbilical cord $\mathrm{Hb}$ level, postnatal 96th hour $\mathrm{Hb}$, serum total and direct bilirubin levels measured with the colorimetric method (diazotized sulfanilic acid reaction, Roche Diagnostics $\mathrm{GmbH}$, Mannheim, Germany), postnatal TcB measurements at 6th, 48th, 96th and 168th hours, and the presence of phototherapy requirement were recorded. The diagnosis of significant hyperbilirubinemia was made considering 
gestational and postnatal age, and risk factors of the newborns ${ }^{6,7}$, and these cases were put on phototherapy. Phototherapy treatment was performed with high-intensity special blue light LED devices emitting $\geq 40 \mu \mathrm{W} / \mathrm{cm}^{2} / \mathrm{nm}$ irradiance from a $45-\mathrm{cm}$ distance for 24 hours.

$\mathrm{TcB}$ measurements were made with a trancutaneous bilirubinometer (Bilichek, Respironics Inc, Monroeville, PA, USA). To take a measurement with this trancutaneous bilirubinometer, the probe was positioned on the infant's skin, and five individual scans were taken to produce one measurement that was displayed in $\mathrm{mg} / \mathrm{dL}$. If an erroneous measurement is taken, an error message is displayed, and the scan should be repeated. The TcB measurements were performed on the sternum/thoracic region. The sternum/thoracic region was not exposed to direct sunlight, and environmental lighting was constant during the study period.

The study was conducted according to clinical practice guidelines and approved by the local ethics committee (IRB number: 18052016-4). Informed consent was obtained from either parent of each patient.

For statistical analysis of the data Statistical Package for Social Sciences (SPSS) 18.0 program was used. Shapiro-Wilk test was used in determining normal distribution. Fisher's Exact $\mathrm{x}^{2}$ test was used to compare the nominal values such as gender, route of delivery, weight loss, number of late preterm and term newborns and whether there was a need of phototherapy treatment between the groups. Of the continuous variable parameters, gestational age and serum direct bilirubin levels were compared with $\mathrm{T}$ test, and maternal age, birth weight, $\mathrm{STB}, \mathrm{Hb}$ and weight loss were compared with Independent Sample T test. Pearson's test was used in analysis of the correlation between the time of cord clamping and the other parameters. Values were given as mean \pm standard deviation and median (25-75\%) range. A p value of $\leq 0.05$ was considered as statistically significant.

A power analysis revealed that to achieve $80 \%$ power to detect a $2 \mathrm{mg} / \mathrm{dl}$ difference in transcutaneous bilirubin levels between groups using a p-value of $0.05,80$ patients would be needed in each group.

\section{Results}

The study was completed with a total of 172 newborns (82 and 90 in Group I and Group II, respectively). Demographic data of the study groups, and comparison of the cord clamping time, cord $\mathrm{Hb}$, postnatal $\mathrm{TcB}$ measurements at 6th, 48th, 96th and 168th hours, 96th hour $\mathrm{Hb}$,

Table I. Demographic features of the patients.

\begin{tabular}{lccc}
\hline Parameter & Group I (n=82) & Group II (n=90) & p value \\
\hline Birth weight (gram)* & $3264 \pm 420(2420-4295)$ & $3282 \pm 416(2140-4190)$ & 0.771 \\
Gestational age (weeks)* & $38.23 \pm 1.05(36-40)$ & $38.37 \pm 1.17(35-41)$ & 0.429 \\
Number of late preterm/term newborns & $15 / 67$ & $16 / 74$ & 0.9301 \\
Apgar score at 1st minute** & $9(8-9)$ & $9(8-9)$ & 0.438 \\
Apgar score at 5th minute** & $9(8-9)$ & $9(8-9)$ & 0.438 \\
Maternal age (years)* & $29.4 \pm 4.83(19-40)$ & $31.1 \pm 4.2(21-44)$ & 0.18 \\
Route of delivery (cesarean/vaginal) & $71 / 11$ & $70 / 20$ & 0.133 \\
Gender (male/female) & $46 / 36$ & $39 / 51$ & 0.94 \\
Need of a phototherapy treatment (yes/no) & $25 / 57$ & $9 / 81$ & 0.001 \\
Weight loss until postnatal 4th day (<5\%/5-9.99\%) & $69 / 21$ & $61 / 21$ & 0.728 \\
Weight loss until postnatal 4th day (gram)* & $160 \pm 14(112-201)$ & $164 \pm 15(113-207)$ & 0.073 \\
*: Values are given as mean \pm standart deviation (minimum-maximum) & &
\end{tabular}


Table II. Cord clamping time and transcutaneous and serum total bilirubin values of the patients.

\begin{tabular}{lccc}
\hline Parameter & Group I $(\mathrm{n}=82)$ & Group II $(\mathrm{n}=90)$ & $\mathrm{p}$ value \\
\hline Cord clamping time (seconds)** & $67(63-69.1)$ & $11(9-14)$ & $<0.001$ \\
Cord blood hemoglobin $(\mathrm{g} / \mathrm{dl})^{*}$ & $17.17 \pm 1.59(12.6-22.2)$ & $14.57 \pm 1.83(8.7-20.1)$ & $<0.001$ \\
Transcutaneous bilirubin at 6th hour $(\mathrm{mg} / \mathrm{dl})^{*}$ & $4.43 \pm 1.01(2-6.3)$ & $4.19 \pm 1.15(1.7-6.9)$ & 0.151 \\
Transcutaneous bilirubin at 48th hour $(\mathrm{mg} / \mathrm{dl})^{*}$ & $9.73 \pm 2.30(4.9-15.6)$ & $9.04 \pm 2.52(3-14)$ & 0.065 \\
Transcutaneous bilirubin at 96th hour $(\mathrm{mg} / \mathrm{dl})^{*}$ & $14.71 \pm 2.89(6.7-25.0)$ & $11.21 \pm 3.14(3.5-18.1)$ & $<0.001$ \\
Transcutaneous bilirubin at 168th hour $(\mathrm{mg} / \mathrm{dl})^{*}$ & $11.68 \pm 3.35(5.5-22.1)$ & $9.52 \pm 3.27(2.8-19.9)$ & $<0.001$ \\
Venous hemoglobin at 96th hour $(\mathrm{g} / \mathrm{dl})^{*}$ & $17.89 \pm 1.90(12.3-23.2)$ & $16.78 \pm 1.99(13.9-22.9)$ & $<0.001$ \\
Serum total bilirubin at 96th hour $(\mathrm{mg} / \mathrm{dl})^{*}$ & $14.49 \pm 3.39(2.2-25.0)$ & $10.34 \pm 3.78(1.8-20.3)$ & $<0.001$ \\
Serum direct bilirubin at 96th hour $(\mathrm{mg} / \mathrm{dl})^{*}$ & $0.36 \pm 0.1(0.1-0.6)$ & $0.32 \pm 0.1(0.2-0.7)$ & 0.271 \\
\hline
\end{tabular}

*: Values are given as mean \pm standart deviation (minimum-maximum)

$* *$ Values are given as median (Range 25th-75th centile)

STB and direct bilirubin levels between the study groups are given in Table I and Table II.

There were no statistically significant differences between the study groups regarding route of delivery, gender, gestational age, birth weight, maternal age, Apgar scores at 1st and 5th minutes, weight loss, 6th hour and 48th hour TcB measurements (Table I). However, there were statistically significant differences between the study groups regarding cord clamping time, cord blood $\mathrm{Hb}, \mathrm{TcB}$ measurements at 96th and 168th hours, and 96th hour venous $\mathrm{Hb}$ and STB levels. The need of phototherapy requirement was higher in Group I $(n=25)$ when compared to Group II $(n=9)$, and thus the incidence of significant hyperbilirubinemia was higher in newborns whose cord was clamped late $(\mathrm{p}=$ 0.001) (Table II).

Cord blood $\mathrm{Hb}$ level was significantly higher in Group I when compared to Group II (17.17 $\pm 1.59 \mathrm{~g} / \mathrm{dl}$ vs $14.57 \pm 1.83 \mathrm{~g} / \mathrm{dl}, \mathrm{p}<0.001$ ) (Table II) (Fig. 1). Analysis of the relationship between cord blood $\mathrm{Hb}$ level and phototherapy treatment revealed that increase in cord blood $\mathrm{Hb}$ for each $1 \mathrm{gr} / \mathrm{dl}$ caused a 3.94-fold increased risk in the requirement of phototherapy treatment (Odds ratio $=3.94, p=0.0012,95 \%$ confidence interval $(\mathrm{CI})=1.71-9.08)$ (Fig. 1).

Regarding postnatal TcB values, there were no statistically significant differences between the groups on the basis of 6 th and 48th hour TcB values, however 96th and 168th hour TcB levels were significantly higher in Group I when compared to Group II ( $\mathrm{p}<0.001$ and $\mathrm{p}<0.001$, respectively) (Table II) (Fig. 2).

96th hour STB level was significantly higher in Group I when compared to Group II $(\mathrm{p}<0.001)$ (Table II) (Fig. 3).

In all of the study cases $(n=172)$ correlation analysis revealed that cord clamping time showed a statistically significant postitive correlation with both cord blood and 96th hour venous $\mathrm{Hb}$ levels $(\mathrm{p}<0.0001, \mathrm{r}=0.426,95 \% \mathrm{CI}=$ $0.291-0.544$ and $\mathrm{p}=0.0002, \mathrm{r}=0.291,95 \% \mathrm{CI}=$ $0.144-0.426$, respectively). Cord clamping time also showed a statistically significant postitive correlation with both 96th hour and 168th hour TcB levels $(p<0.0001, r=0.307,95 \%$ CI $=0.161$ 0.440 and $p=0.0009, r=0.257,95 \%$ CI $=0.108$ 0.395). There was a statistically significant postitive correlation between cord clamping

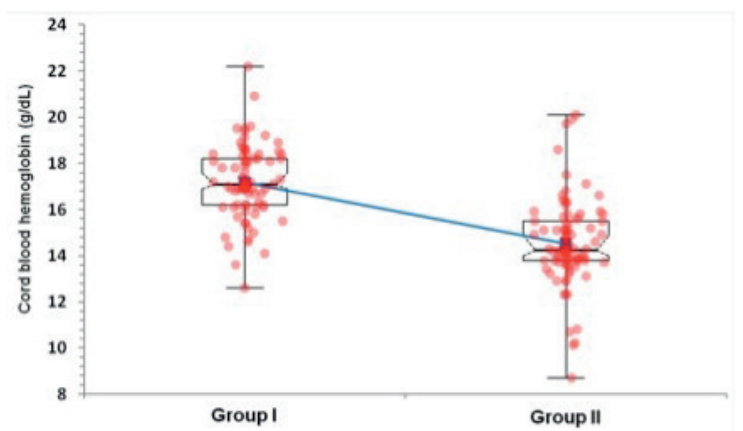

Fig. 1. Comparison of the cord blood hemoglobin levels between the groups. 


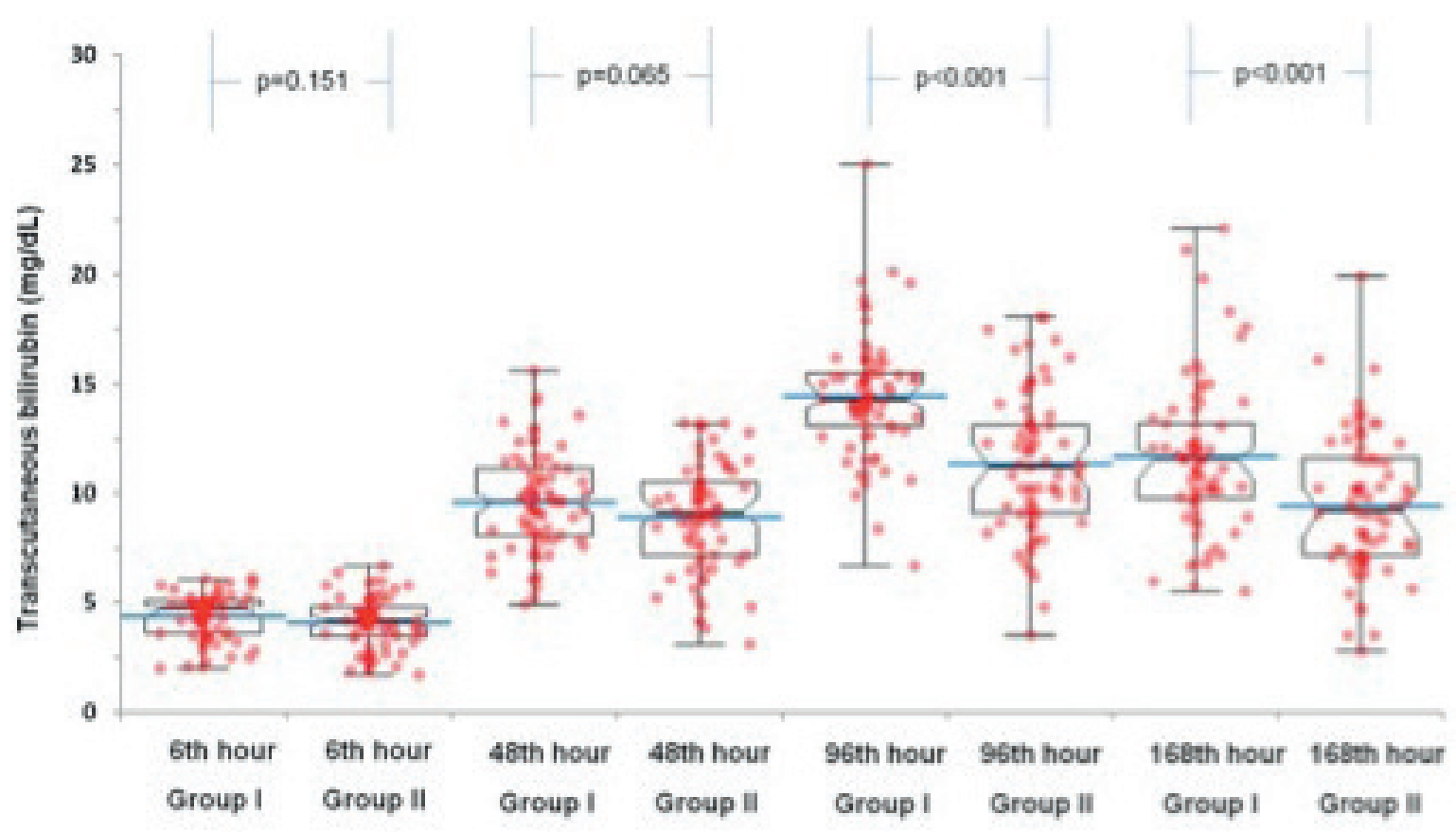

Fig. 2. Comparison of the 6 th, 48 th, 96 th and 168 th hour transcutaneous bilirubin values between the groups.

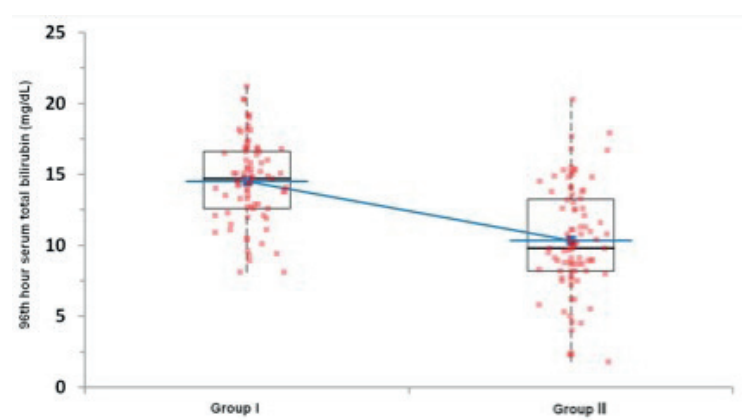

Fig. 3. Comparison of the 96th hour serum total bilirubin levels between the study groups.

time and 96th hour STB levels ( $\mathrm{p}<0.0001$, $\mathrm{r}=$ $0.310,95 \% \mathrm{CI}=0.165-0.443)$.

\section{Discussion}

Although the relationship between umbilical cord clamping time and various parameters such as postnatal and early infancy $\mathrm{Hb}$ levels, iron deficiency, iron deficiency anemia and risk of neonatal jaundice requiring phototherapy treatment has previously been studied ${ }^{5,8-11}$, to the best of our knowledge, there have been no studies investigating the relationship between cord clamping time and the risk of significant hyperbilirubinemia by serial longitudinal TcB measurements, and early postnatal $\mathrm{Hb}$ and STB measurements. This study is the first in this field in this regard.

Early cord clamping is defined as clamping the cord in 60 seconds after delivery of the baby, and late cord clamping is defined as clamping the cord when the pulsation stops or at least 60 seconds or more after delivery of the baby in the metaanalytic Cochrane study..$^{12}$ Mercer et al. ${ }^{9}$ have reported that late cord clamping increases neonatal $\mathrm{Hb}$ levels at birth and gains time for transfer of blood from placenta to newborn. This placental transfusion provides the newborn an additional $30 \%$ blood volume and an additional erythrocyte volume up to $60 \% .^{2-4}$ This increased placental transfusion provides a higher neonatal $\mathrm{Hb}$ concentration and an increased erythrocyte flux to vital organs, a better neonatal cardiopulmonary adaptation, a higher incidence of successful breast-feeding, and better body iron stores and fewer iron deficiency anemia. ${ }^{3,4}$ On the other hand early cord clamping increases feto-maternal transfusion and a great amount of blood remains in placenta. ${ }^{12}$ Nesheli et al. ${ }^{10}$ 
compared early versus late cord clamping in 66 newborns, and reported higher neonatal $\mathrm{Hb}$ and hematocrit levels and better serum iron status at 6 months of age in newborns whose cord was clamped late. They defined high neonatal $\mathrm{Hb}$ levels as a predisposing risk factor for polycytemia and hyperbilirubinemia. Mercer et al. ${ }^{9}$ also compared early versus late ( $>5$ minutes) cord clamping with respect to venous $\mathrm{Hb}$ and STB levels at 24th and 48th hours of life, and reported no statistically significant difference in STB levels between the two groups despite higher $\mathrm{Hb}$ levels in those whose cord is clamped early.

In the single study investigating the relationship between umbilical cord $\mathrm{Hb}$ level and the risk of neonatal hyperbilirubinemia, higher cord $\mathrm{Hb}$ levels were observed with increasing cord clamping time, and both were associated with an increased risk of significant hyperbilirubinemia requiring phototherapy treatment. ${ }^{5}$ However umbilical cords of all the cases were clamped in the first 15 seconds after delivery without constituting early or late clamping groups in that study. ${ }^{5}$ In the cases receiving and not receiving phototherapy, mean $\mathrm{Hb}$ level was $17.4 \mathrm{~g} / \mathrm{dl}$ and $15.8 \mathrm{~g} / \mathrm{dl}$, respectively, and the authors did not recommend late cord clamping for their country. ${ }^{5}$ In our study the two groups were composed according to the criteria of early and late clamping, and mean cord $\mathrm{Hb}$ levels were $14.57 \mathrm{~g} / \mathrm{dl}$ and $17.17 \mathrm{~g} / \mathrm{dl}$ in the groups of early and late clamping, respectively. In the study by Nakagawa et al. ${ }^{5}$ cord blood $\mathrm{Hb}$ level determined the phototherapy requirement with an Odds ratio of 1.74 . Odds ratio in our study was 3.94 indicating that late clamping of the cord increased the risk of significant hyperbilirubinemia by 3.94 -fold.

Chien et al. ${ }^{13}$ compared early ( $<60 \mathrm{sec}$ ) versus late $(>180 \mathrm{sec})$ cord clamping in 105 newborns. They followed the newborns with respect to significant hyperbilirubinemia postnatally for 4 to 7 days after hospital discharge, and no significant difference was observed between the early and late clamping groups. Yang et al. ${ }^{14}$ compared two eras of a delayed cord-clamping protocol (before and after) for term neonates and reported significantly higher transcutaneous bilirubin levels, an increased number of serum blood draws, and more clinical diagnoses of jaundice. However, they reported no increase in the incidence of phototherapy. In our study both 96th hour STB and TcB levels and 168th hour TcB levels were significantly higher in newborns whose cord was clamped late. Higher bilirubin levels on day 4 and after and significantly higher phototherapy requirement in the end in these newborns may be explained by both the combined effects of the degradation of high $\mathrm{Hb}$ load taken over from the umbilical cord (Late clamping) and transformation of $\mathrm{Hb}$ to Heme in these days (Physiologic jaundice).

In the Cochrane meta-analysis of 15 different studies, the ratio of newborns receiving phototherapy was higher ( $4.36 \%$ vs $2.74 \%)$ and mean $\mathrm{Hb}$ concentration at 24th-48th hours of life was higher in newborns whose cord was clamped late. ${ }^{12}$ Mercer et al. ${ }^{9}$ reported higher $\mathrm{Hb}$ levels at 24th-48th hours of life in newborns whose cord was clamped late $(>5 \mathrm{~min})$ when compared to those whose cord was clamped early (19.4 g/dl vs $17.8 \mathrm{~g} / \mathrm{dl})$. In our study mean 96th hour $\mathrm{Hb}$ level was statistically significantly higher in cases whose cord was clamped late in comparison to those whose cord was clamped early $(17.8 \mathrm{~g} / \mathrm{dl}$ vs $16.7 \mathrm{~g} / \mathrm{dl})$. According to the Cochrane meta-analysis and recommendations of the American College of Obstetricians and Gynecologists, potential harms of increased $\mathrm{Hb}$ should be evaluated by clinicians considering the region they worked and the accessibility to facilities of jaundice diagnosis and treatment. ${ }^{12,15}$ For example late cord clamping should be less preferred in regions where the treatment of neonatal jaundice is not easily accessible and untreated significant hyperbilirubinemia has the risks of long-term complications. ${ }^{12,15}$

In this study, we investigated the relationship between umbilical cord clamping time, and transcutaneous bilirubin levels and the risk of neonatal significant hyperbilirubinemia, and demonstrated that late cord clamping caused higher postnatal $\mathrm{Hb}$ and bilirubin $(\mathrm{TcB}$ and 
STB) levels, and a higher incidence of significant hyperbilirubinemia requiring phototherapy treatment in comparison to early cord clamping. Although positive effects of late cord clamping on neonatal $\mathrm{Hb}$ levels and body store iron status in late infancy have been demonstrated in previous studies, newborns whose cord are clamped late should be followed up closely with respect to high postnatal bilirubin levels and other risks associated with significant hyperbilirubinemia requiring phototherapy treatment.

\section{REFERENCES}

1. Wyllie J, Bruinenberg J, Roehr CC, Rüdiger M, Trevisanuto D, Urlesberger B. European Resuscitation Council Guidelines for Resuscitation 2015: section 7. resuscitation and support of transition of babies at birth. Resuscitation 2015; 95: 249-263.

2. McDonald S. Physiology and management of the third stage of labour. In: Fraser DM, Cooper MA, (eds). Myles Textbook for Midwives. (14th ed) Edinburgh: Churchill Livingstone, 2003.

3. Mercer JS. Current best evidence: a review of the literature on umbilical cord clamping. J Midwifery Womens Health 2001; 46: 402-414.

4. Palethorpe RJ, Farrar D, Duley L. Alternative positions for the baby at birth before clamping the umbilical cord. Cochrane Database Syst Rev 2010; 10: CD007555.

5. Nakagawa M, Ishida $Y$, Nagaoki $Y$, et al. Correlation between umbilical cord hemoglobin and rate of jaundice requiring phototherapy in healthy newborns. Pediatr Int 2015; 57: 626-628.

6. American Academy of Pediatrics Subcommittee on Hyperbilirubinemia. Management of hyperbilirubinemia in the newborn infant 35 or more weeks of gestation. Pediatrics 2004; 114: 297316.
7. Maisels MJ, Bhutani VK, Bogen D, Newman TB, Stark AR, Watchko JF. Hyperbilirubinemia in the newborn infant $\geq 35$ weeks' gestation: an update with clarifications. Pediatrics 2009; 124: 1193-1198.

8. Ceriani Cernadas JM, Carroli G, Pellegrini L, et al. The effect of timing of cord clamping on neonatal venous hematocrit values and clinical outcome at term: a randomized, controlled trial. Pediatrics 2006; 117: e779-e786.

9. Mercer JS, Erickson-Owens DA, Collins J, Barelos MO, Parker AB, Padbury JF. Effects of delayed cord clamping on residual placental blood volume, hemoglobin and bilirubin levels in term infants: a randomized controlled trial. J Perinatol 2017; 37: 260-264.

10. Nesheli HM, Esmailzadeh S, Haghshenas M, Bijani A, Moghaddams TG. Effect of late vs early clamping of the umbilical cord (on hemoglobin level) in fullterm neonates. J Pak Med Assoc 2014; 64: 1303-1305.

11. McDonald SJ, Middleton P, Dowswell T, Morris PS Effect of timing of umbilical cord clamping of term infants on maternal and neonatal outcomes. Evid Based Child Health 2014; 9: 303-397.

12. Rabe H, Diaz-Rossello J, Duley L, Dowswell T. Effect of timing of umbilical cord clamping and other strategies to influence placental transfusion at preterm birth on maternal and infant outcomes. Cochrane Database Syst Rev 2012; 8: CD003248.

13. Chien PC, Yang CC, Gau ML, Liu CY, Lee TY. The impact of late umbilical cord clamping on neonatal jaundice and postpartum hemorrhage: a randomized controlled trial. Hu Li Za Zhi 2015: 62; 41-53.

14. Yang S, Duffy JY, Johnston R, Fall C, Fitzmaurice LE. Association of a delayed cord-clamping protocol with hyperbilirubinemia in term neonates. Obstet Gynecol 2019; 133: 754-761.

15. Committee on Obstetric Practice. Delayed umbilical cord clamping after birth. Committee Opinion. The American College of Obstetricians and Gynecologists. Obstetr Gynecol 2017; 129: e5-e10. 